\title{
Dynamic regulation of murine cortical transcriptome by early-life stress: Impairment of myelination and cognitive functions
}

\author{
Natalya Bondar \\ ICG SB RAS, Novosibirsk, Russia \\ nbondar@bionet.nsc.ru \\ Nikita Ershov \\ ICG SB RAS, Novosibirsk, Russia \\ ershov@bionet.nsc.ru
}

\author{
Anastasia Shulyupova \\ ICG SB RAS, Novosibirsk, Russia \\ shulyupova@bionet.nsc.ru \\ Elena Antontseva \\ ICG SB RAS, Novosibirsk, Russia \\ antontseva@bionet.nsc.ru
}

\author{
Polina Kisaretova \\ ICG SB RAS, Novosibirsk, Russia \\ kisaretova@bionet.nsc.ru \\ Tatiana Merkulova \\ ICG SB RAS, Novosibirsk, Russia \\ merkulova@bionet.nsc.ru
}

\begin{abstract}
Stress early in life negatively affects the formation of the brain regions. We explored the effects of two types of stress in mice: prolonged repeated separation of pups from their mothers for $3 \mathrm{~h}$ per day during the first 2 weeks of life (maternal separation, MS), or $24 \mathrm{~h}$ single maternal separation on 9th day of life (maternal deprivation, MD). RNA-seq analysis revealed weak transcriptome changes in prefrontal cortex of 15day pups but at the same time had strong delayed consequences on gene expression in adult mice. The observed changes were mainly related to genes associated with myelin sheath development. The direction of the alterations in 15-day pups and adult mice were mainly opposite.
\end{abstract}

Keywords - early life stress, RNA-seq, myelin, mice.

Motivation and Aim

Stressful events in early postnatal period have critical consequences for the individuals' life and can increase later risk for the development of psychiatric disorders. The maturation of brain structures continues in the postnatal period in both humans and animals. Early-life stress negatively affects the formation of the brain regions taking part in the implementation of emotion, memory and cognition. Various animal models have been developed to investigate the molecular consequences of early life stressful events. Among them, maternal separation models in rodents are the most commonly used and established ones [1]. Prolonged maternal separation causes significant amounts of stress resulting in negative long-lasting changes of emotion-related behavior, stress reactivity and cognitive functions in rodents [2-5].

The aim of our study was the investigation of the transcriptome profile in the prefrontal cortex both immediately after prolonged maternal separation and through a long period of comfortable life. Comparison of immediate and delayed effects on transcription should contribute significantly to our knowledge of a link between early life exposures and neurobiological and behavioral outcomes in adulthood. Given the fact that the prefrontal cortex is responsible for complex cognitive functions, regulation of emotion, and adaptation to stress, we expected to reveal some alterations in gene expression in this brain region reflecting the long-lasting consequences of stress early in life.

\section{Methods}

We used two types of early life stress: prolonged repeated separation of pups from their mothers for $3 \mathrm{~h}$ per day during the first 2 weeks of life (maternal separation, MS), or $24 \mathrm{~h}$ single maternal separation on 9th day of life (maternal deprivation, MD). We analyzed the effects of stress on transcriptome of the prefrontal cortex of 15-days pups (at $24 \mathrm{~h}$ after the last stress exposure) and 3 months old adult mice. To validate the RNA-seq results, the expression of selected up- or down-regulated genes were confirmed by real-time PCR.

\section{Results}

We revealed the weak effect of stress on the expression patterns of genes in 15-days pups: only 6 genes were differentially expressed in MS group to compare with controls. Stress early in life resulted in changes in expression of genes whose proteins connected with myelin sheath development (Mag, Mobp, Mog, Mal, Plpl and Ugt8a, all down-regulated). Despite weak changes in pups, transcriptome analysis of the prefrontal cortex in adult mice showed a number of differentially expressed genes in MS and MD groups compared to controls (648 genes in MS group and 192 genes in MD group). In MS group the changes mainly occur in systems associated with the development of the myelin sheath, regulation of trans-synaptic signaling and regulation of membrane potential. All the genes that were changed in prefrontal cortex of 15-days pups also remained altered in adults, but in the opposite direction (Fig. 1). Genes, which were differentially expressed in MD group to compare with controls, relate to different systems, without significant overrepresentation of any one.

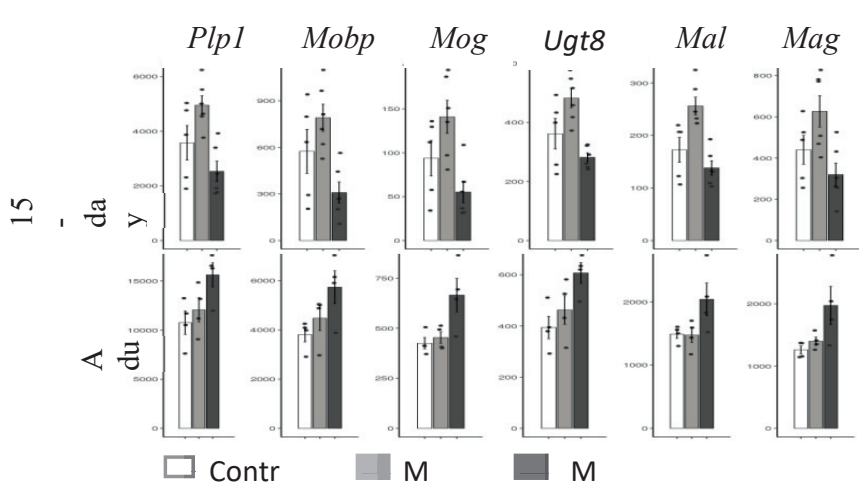

Fig. 1. Level of expression Plp1, Mobp, Mog, Ugt8a, Mal and Mag genes in adult mice and 15-day pups with history of earlylife stress. Data represent as normalized counts. 
The obtained results suggest the existence of delayed longterm expression changes in the prefrontal cortex triggered by early postnatal stress. We can suggest that disturbances in myelination can underlie enhanced susceptibility to mental illness.

\section{ACKNOWLEDGMENT}

The work was supported by Russian Science Foundation project 16-15-10131.

\section{REFERENCES}

[1] Pryce C.R., Feldon J. Long-term neurobehavioural impact of the postnatal environment in rats: manipulations, effects and mediating mechanisms. Neurosci Biobehav Rev. 2003. 27(1-2): 57-71.
[2] Bondar N.P., Lepeshko A.A., Reshetnikov V.V. Effects of early-life stress on social and anxiety-like behaviors in adult mice: sex-specific effects. Behav Neurol. 2018. 2018: 1538931.

[3] Di Segni M., Andolina D., Ventura R. Long-term effects of early environment on the brain: Lesson from rodent models. Semin Cell Dev Biol. 2018. 77: 81-92.

[4] Franklin T.B., Saab B.J., Mansuy I.M. Neural mechanisms of stress resilience and vulnerability. Neuron. 2012. 75(5): 747-761.

[5] Reshetnikov V.V., Studenikina A.A., Ryabushkina J.A., Merkulova T.I., Bondar N.P. The impact of early-life stress on the expression of HPA-associated genes in the adult murine brain. Behaviour. 2018. 155(2-3): 181 - 203. 